\title{
Quality Assurance for Respiratory-Gated Radiotherapy Using the Real-Time Tumor-Tracking Radiotherapy System
}

\author{
Takehiro Shiinoki ${ }^{*}$, Shinji Kawamura ${ }^{2}$, Takuya Uehara², Yuki Yuasa², Takeshi Kamomae ${ }^{3}$, \\ Takaya Kotakebayashi'2, Masahiro Koike², Ryuji Kanzaki², Sung Chul Park', \\ Hideki Hanazawa', Shotaro Takahashi', Keiko Shibuya ${ }^{1}$ \\ ${ }^{1}$ Department of Therapeutic Radiology, Graduate School of Medicine, Yamaguchi University, Yamaguchi, Japan \\ ${ }^{2}$ Department of Radiological Technology, Yamaguchi University Hospital, Yamaguchi, Japan \\ ${ }^{3}$ Department of Therapeutic Radiology, Graduate School of Medicine, Nagoya University, Aichi, Japan \\ Email: shiinoki@yamaguchi-u.ac.jp
}

Received 20 May 2014; revised 28 June 2014; accepted 18 July 2014

Copyright (C) 2014 by authors and Scientific Research Publishing Inc.

This work is licensed under the Creative Commons Attribution International License (CC BY).

http://creativecommons.org/licenses/by/4.0/

c) (i) Open Access

\section{Abstract}

Purpose: Respiratory-gated radiation therapy (RT) using the real-time tumor-tracking radiotherapy (RTRT) system is an effective technique for managing tumor motion. High dosimetric and geometric accuracy is needed; however, quality assurance (QA) for respiratory-gated RT using the RTRT system has not been reported. The purpose of this study was to perform QA for respiratorygated RT using the RTRT system. Materials and Methods: The RTRT system detected the position of the fiducial marker and radiation delivery gated to the motion of the marker was performed. The dynamic anthropomorphic thorax phantom was positioned at the isocenter using the fiducial marker in the phantom. The phantom was irradiated only when the fiducial marker was within a three-dimensional gating window of $\pm 2 \mathrm{~mm}$ from the planned position. First, the absolute doses were measured using anionization chamber inserted in the phantom under the stationary, gating and non-gating state for sinusoidal (nadir-to-peak amplitude [A]: 20 - $40 \mathrm{~mm}$, breathing period [T]: 2 - 4 s) and the basic respiratory patterns. Second, the dose profiles were measured using Gafchromic films in the phantom under the same conditions. Differences between dose profiles were calculated to evaluate the dosimetric and geometric accuracy. Finally, differences between the actual and measured position of the fiducial marker were calculated to evaluate the tracking accuracy for sinusoidal and basic respiratory patterns. Results: For the sinusoidal patterns, the relative doses were 0.93 for non-gating and 0.99 for gating $(A=20 \mathrm{~mm}, T=2 \mathrm{~s}), 0.94$ for non-gating and 1.00 for gating $(A=20 \mathrm{~mm}, T=4 \mathrm{~s}), 0.55$ for non-gating and 1.00 for gating $(A=40 \mathrm{~mm}, T=4$

\footnotetext{
"Corresponding Author. Presented in part at the 13th Annual Meeting of the Asia-Oceania Congress of Medical Physics (AOCMP), Singapore, 12-14 December 2013. 
s), respectively. For the basic respiratory pattern, the relative doses were 1.00 for non-gating and 1.00 for gating, respectively. Compared to the stationary conditions, the differences in lateral distance between the $90 \%$ dose of dose profiles were $6.23 \mathrm{~mm}$ for non-gating and $0.36 \mathrm{~mm}$ for gating $(A=20 \mathrm{~mm}, T=2 \mathrm{~s}), 8.79 \mathrm{~mm}$ for non-gating and $1.73 \mathrm{~mm}$ for gating $(A=20 \mathrm{~mm}, T=4 \mathrm{~s}), 18.37$ $\mathrm{mm}$ for non-gating and $0.67 \mathrm{~mm}$ for gating $(A=40 \mathrm{~mm}, \mathrm{~T}=4 \mathrm{~s})$, respectively. For the basic respiratory pattern, those were $5.23 \mathrm{~mm}$ for non-gating and $0.35 \mathrm{~mm}$ for gating. The root mean square (RMS) values of the tracking error were $0.18 \mathrm{~mm}(A=20 \mathrm{~mm}, T=2 \mathrm{~s}), 0.14 \mathrm{~mm}(A=20 \mathrm{~mm}, T=4$ s), and $0.21 \mathrm{~mm}(A=40 \mathrm{~mm}, T=4 \mathrm{~s})$ for sinusoidal and $0.79 \mathrm{~mm}$ for the basic respiratory pattern, respectively. Conclusion: We conducted QA for respiratory-gated RT using the RTRT system. The respiratory-gated RT using the RTRT system reduced the blurring effects on dose distribution with high dosimetric and geometric accuracy.

\section{Keywords}

\section{Quality Assurance, Respiratory Gating, Real-Time Tumor-Tracking Radiotherapy System}

\section{Introduction}

In radiation therapy (RT), tumor motion during respiration results in significant geometric and dosimetric uncertainties in the dose delivery to the thorax and abdomen. Conventionally, large internal margins (IMs) are needed to fully cover the geometric changes that occur during free breathing; these large IMs may result in toxicity to healthy tissue. As techniques for managing respiratory-induced tumor movement, breath-holding, respiratory-gated RT, and four-dimensional techniques are effective in reducing the IM, resulting in a lower dose to the normal tissue and thus a lower risk of complications [1]-[6].

The real-time tumor-tracking radiotherapy (RTRT) system (Mitsubishi Electronics Co., Ltd., Tokyo) consists of two sets of diagnostic X-ray TV systems that are mounted in the treatment room. Using X-ray tubes from two directions, the position of the fiducial marker displayed on the X-ray monitor is automatically extracted using a pattern recognition technique to calculate the coordinates. When the fiducial marker comes within several millimeters of the planned position, the beam is delivered [6].

Several investigators have reported the quality assurance (QA) for respiratory-gated RT using an external signal [7] [8]. Our group has aimed to perform the respiratory-gated RT using the fiducial marker for lung cancer with high accuracy. Therefore, it is important to perform QA of the RTRT system.

The task group (TG) for the QA of medical accelerators was constituted by the American Association of Physicists in Medicine (AAPM) [9]. The QA of image-guided radiotherapy devices and medical accelerators has been reported. However, the QA of the RTRT system has not been reported. High dosimetric and geometric accuracy is needed for respiratory-gated RT using the RTRT system.

The purpose of this study was to perform QA for respiratory-gated RT using the RTRT system. First, the chamber measurements were performed using both gating and non-gating for moving the dynamic anthropomorphic thorax phantom with the RTRT system. Subsequently, the dose profiles were measured in the same matter using Gafchromic films. Finally, the differences between the actual and measured position of the fiducial marker were calculated to evaluate the tracking accuracy.

\section{Materials and Methods}

\subsection{Computed Tomography Acquisition and Treatment Planning for the Phantom}

For treatment planning, the dynamic anthropomorphic thorax phantom (CIRS. Inc., Norfolk, VA) that had ionization chamber or film inserts was scanned under the stationary condition with 3.0-mm thickness using a 4-slice computed tomography (CT) scanner (Asteion; Toshiba Medical Systems, Tokyo, Japan) (Figure 1). The ionization chamber insert included a 3-cm soft-tissue-equivalent target. These inserts had several fiducial markers.

The treatment plan was designed using Pinnacle ver. 9.0. (Philips Radiation Oncology Systems, Fitchburg, WI). A single photon beam was set at a gantry angle 0 degree. Afield and multi-leaf collimator were fitted to the 
target. The photon beam energy was set to $6 \mathrm{MV}$ and the prescribed dose was set to $200 \mathrm{cGy}$. The planned position of the fiducial marker was set on the CT images.

\subsection{Respiratory-Gating Delivery and Experimental Setup}

Measurements were performed on a MHCL-15DP linear accelerator (Mitsubishi Electronics Co., Ltd., Tokyo), which has a 120-leaf independently moving MLC with 5-mm leaf width at the isocenter of the 20-cm central field. The experimental setup is shown in Figure 2. The RTRT system detected the position of the fiducialmarker and radiation delivery gated to the motion of the marker was performed. The dynamic anthropomorphic thorax phantom was positioned at the isocenter using the fiducial marker in the insert. The phantom was irradiated only when the fiducialmarker was within a three-dimensional gating window of $\pm 2 \mathrm{~mm}$ from the planned position. The dynamic anthropomorphic thorax phantom was driven in the superior-inferior (SI) direction using sinusoidal (nadir-to-peak amplitude [A]: 20 - $40 \mathrm{~mm}$, breathing period [T]: 2 - 4 s) and the basic respiratory patterns. The driving accuracy of the dynamic anthropomorphic thorax phantom was $\pm 0.1 \mathrm{~mm}[10]$.

\subsection{Chamber Measurement}

The measurements were performed using a $0.015 \mathrm{~cm}^{3}$ ionization chamber (PTW31016 pinpoint chamber; PTW, Freiburg GmbH Germany) inserted in the phantom under the following conditions: stationary, gating and nongating for the phantom driven as described above. Dose values were relative to the dose measured for a static
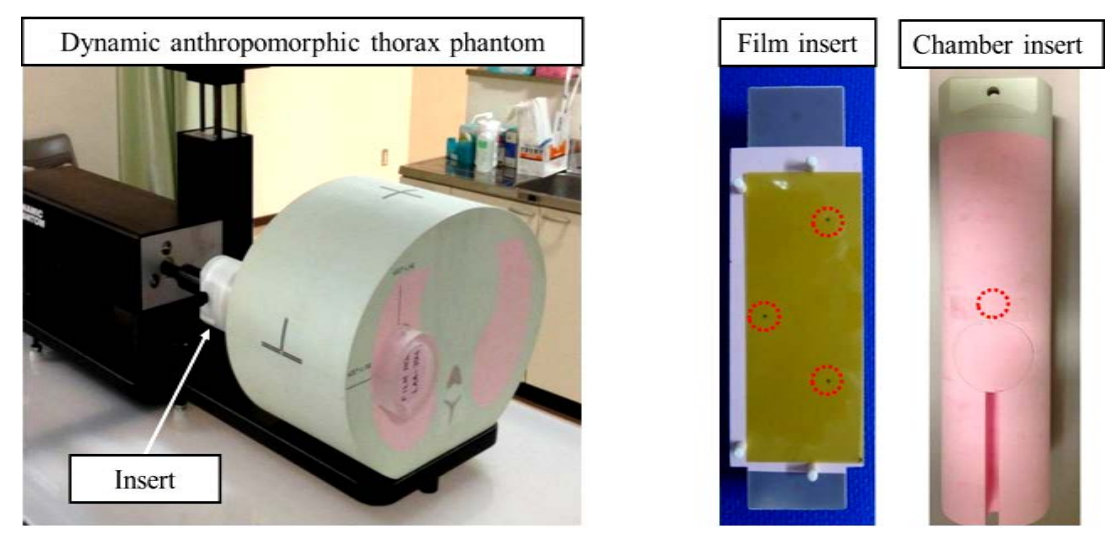

Figure 1. Photographs of a dynamic anthropomorphic thorax phantom (CIRS. Inc., Norfolk, VA) (left) and the film and chamber inserts with several fiducial markers (red circles) (right).

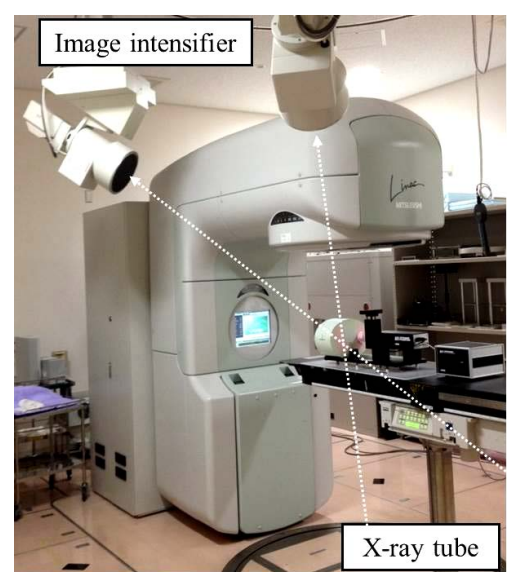

Figure 2. Experimental setup. The dynamic anthropomorphic thorax phantom was positioned at the isocenter. Using X-ray tubes from two directions, the position of the fiducial marker was measured. 
beam to a stationary phantom.

\subsection{Dose Profile Measurement}

Gafchromic EBT3 films (International Specialty Products Corporation, Wayne, NJ) inserted in the phantom were irradiated under the same conditions as the chamber measurement. The irradiated films were scanned in the same orientation (ES-10000G, Seiko Epson Co., Nagano, Japan), with a resolution of 72 dpi in 48-bit color scale with 24-hour post-exposure. The films were analyzed using commercially available radiation dosimetry software (DD systemversion 10.12; R’Tech Inc., Tokyo, Japan). The dose distribution was normalized to the maximum dose using a calibration curve to reduce film uncertainty [11].

The dose profiles perpendicular to the beam axis through the isocenter were measured. The differences between the $90 \%$ dose of the dose profile for non-gating/gating and stationary were calculated to evaluate the dosimetic and geometric accuracy [12].

\subsection{Tracking Accuracy}

The positions of the fiducial marker in the phantom driven using sinusoidal and basic respiratory patterns were measured using the RTRT system and recorded as log files.

The differences between actual and measured positions of the fiducial marker were defined as the tracking error. The root mean square (RMS) values for the tracking error were calculated to evaluate the tracking accuracy.

\section{Results}

\subsection{Chamber Measurement}

The results of chamber measurements are shown in Table 1. Dose values were relative to the dose measured for a static beam to a stationary phantom. Forsinusoidal patterns, the relative doses were 0.93 for non-gating and 0.99 for gating $(\mathrm{A}=20 \mathrm{~mm}, \mathrm{~T}=2 \mathrm{~s}), 0.94$ for non-gating and 1.00 for gating $(\mathrm{A}=20 \mathrm{~mm}, \mathrm{~T}=4 \mathrm{~s}), 0.55$ for non-gating and 1.00 for gating $(A=40 \mathrm{~mm}, \mathrm{~T}=4 \mathrm{~s})$, respectively. For the basic respiratory pattern, the relative doses were 1.00 for non-gating and 1.00 for gating, respectively.

\subsection{Dose Profile Measurement}

Figure 3 shows that an example of the Gafchromic film for the sinusoidal pattern $(A=40 \mathrm{~mm}, \mathrm{~T}=4 \mathrm{~s})$. The respiratory-gated RT using the RTRT system reduced the blurring effects on the dose distribution. Figures 4(a)-(d) show the dose profiles for stationary, non-gating and gating for sinusoidal and the basic respiratory

Table 1. Chamber measurement. The measurements were performed using a $0.015 \mathrm{~cm}^{3}$ ionization chamber inserted in the phantom under the following conditions: stationary, gating and non-gating for the phantom driven as described above. Dose values are relative to dose measured for a static beam to a stationary phantom.

\begin{tabular}{ccc}
\hline Phantom Moving & Beam Gating & Relative Dose \\
\hline Sinusoidal pattern & No & 0.93 \\
$\mathrm{~A}=20 \mathrm{~mm}, \mathrm{~T}=2 \mathrm{~s}$ & On & 0.99 \\
Sinusoidal pattern & No & 0.94 \\
$\mathrm{~A}=20 \mathrm{~mm}, \mathrm{~T}=4 \mathrm{~s}$ & On & 1.00 \\
Sinusoidal pattern & No & 0.55 \\
$\mathrm{~A}=40 \mathrm{~mm}, \mathrm{~T}=4 \mathrm{~s}$ & On & 1.00 \\
Basic respiratory pattern & No & 1.00 \\
(mean A $=15.2 \mathrm{~mm}$, mean $\mathrm{T}=4.8 \mathrm{~s})$ & On & 1.00 \\
\hline
\end{tabular}

Abbreviations: $\mathrm{A}$ = nadir-to-peak amplitude; $\mathrm{T}$ = breathing period. 


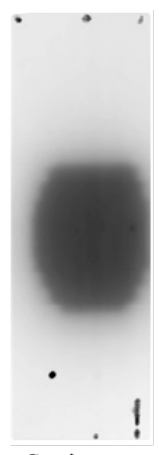

Stationary

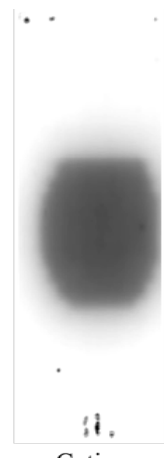

Gating

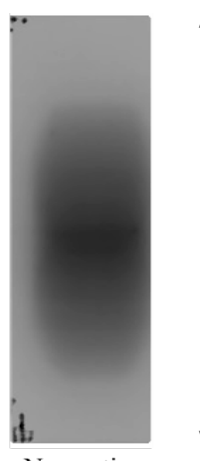

Non-gating
Phantom moving direction

Figure 3. An example of the Gafchromic films for sinusoidal pattern (nadirto-peak amplitude [A]: $40 \mathrm{~mm}$, breath period [T]: $4 \mathrm{~s}$ ). Respiratory-gated RT using the RTRT system reduced the blurring effects on the dose distribution.

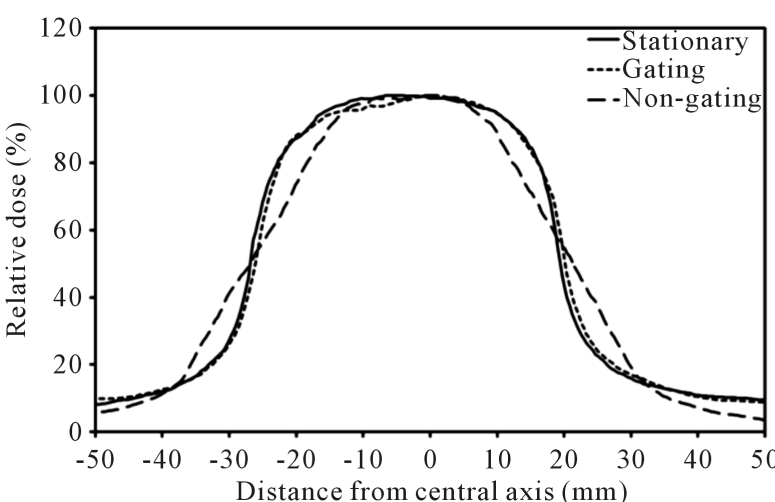

(a)

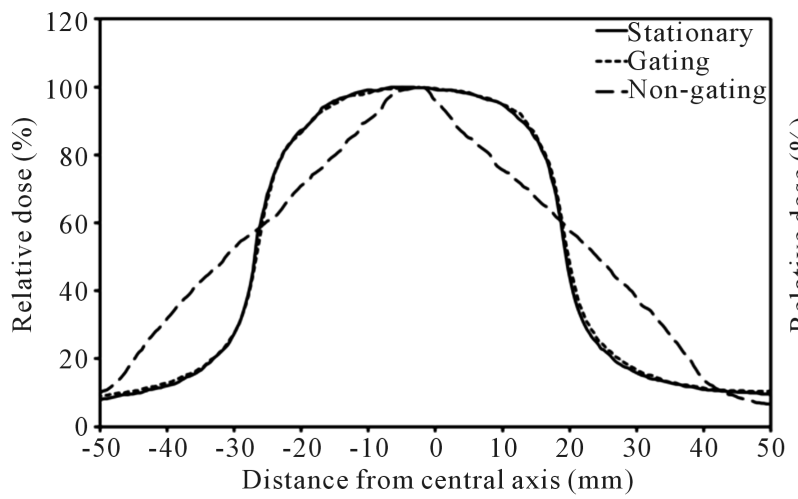

(c)

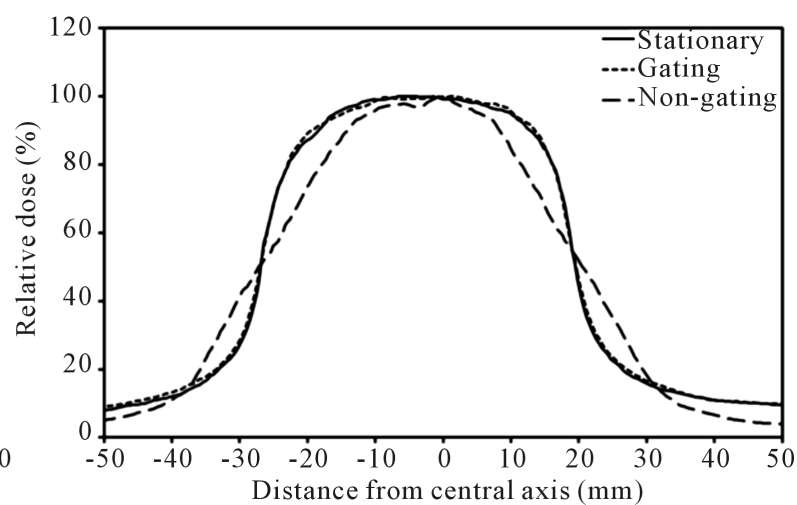

(a)

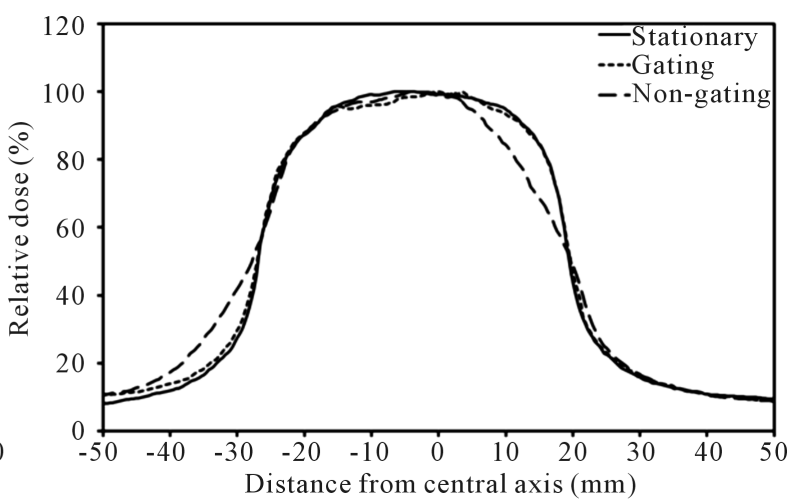

(d)

Figure 4. The dose profiles for stationary, non-gating and gating for sinusoidal patterns (a) $\mathrm{A}=20 \mathrm{~mm}, \mathrm{~T}=2 \mathrm{~s}$; (b) $\mathrm{A}=20$ $\mathrm{mm}, \mathrm{T}=4 \mathrm{~s}$; (c) $\mathrm{A}=40 \mathrm{~mm}, \mathrm{~T}=4 \mathrm{~s}$; and (d) the basic respiratory pattern (mean $\mathrm{A}=15.2 \mathrm{~mm}$, mean $\mathrm{T}=4.8 \mathrm{~s}$ ).

patterns. Compared to the stationary conditions, the differences in lateral distance between the $90 \%$ dose of dose profiles were $6.23 \mathrm{~mm}$ for non-gating and $0.36 \mathrm{~mm}$ for gating (Figure 4(a)), $8.79 \mathrm{~mm}$ for non-gating and 1.73 $\mathrm{mm}$ for gating (Figure 4(b)), $18.37 \mathrm{~mm}$ for non-gating and $0.67 \mathrm{~mm}$ for gating (Figure 4(c)), respectively. For basic respiratory pattern, those were $5.23 \mathrm{~mm}$ for non-gating and $0.35 \mathrm{~mm}$ for gating (Figure 4(d)).

\subsection{Tracking Accuracy}

Figures 5(a)-(d) show the variations in the actual and measured positions of the fiducial marker for the sinusoidal pattern and basic respiratory patterns. The RMS values of the tracking error were $0.18 \mathrm{~mm}(\mathrm{~A}=20 \mathrm{~mm}, \mathrm{~T}$ 

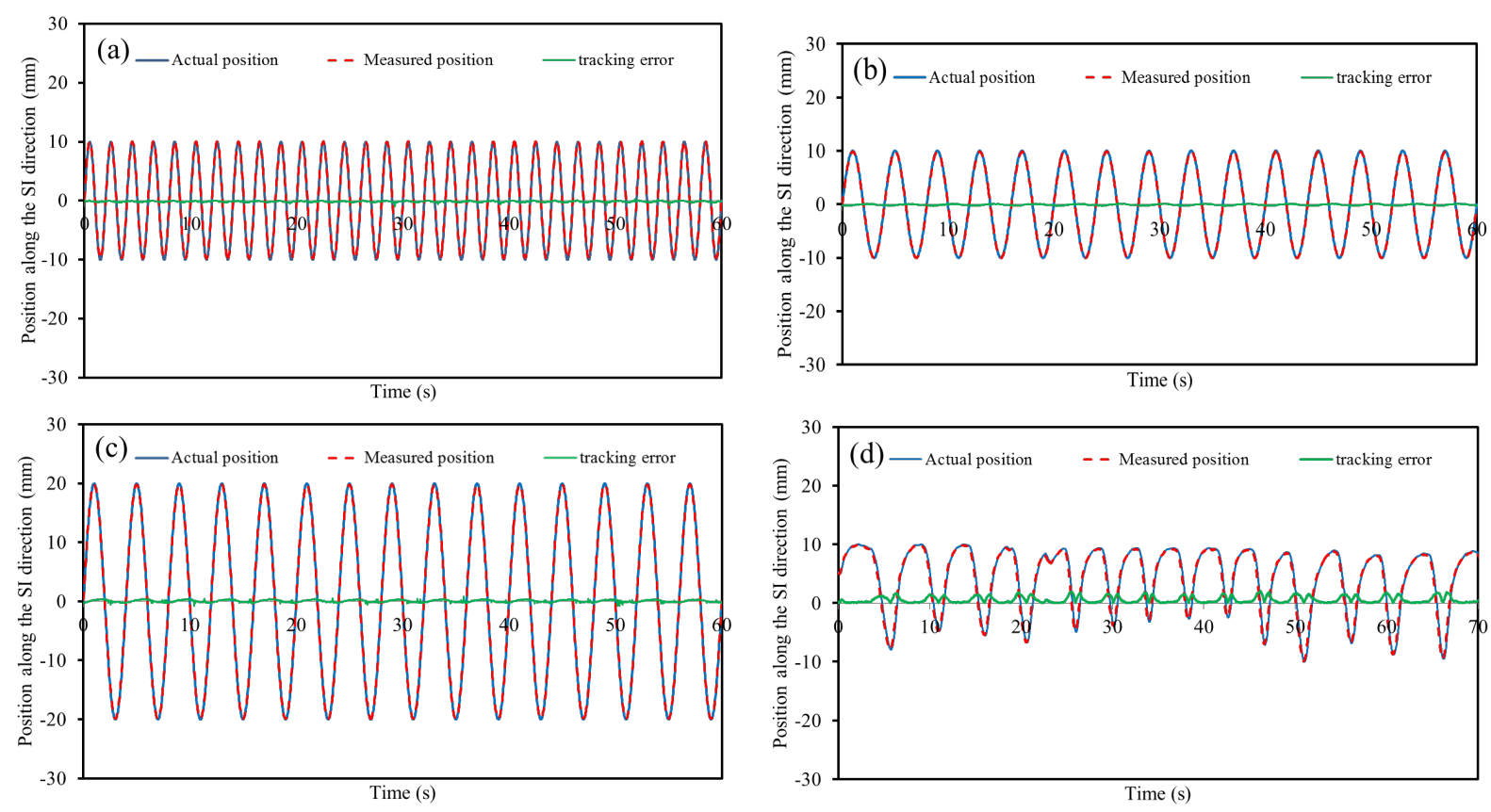

Figure 5. Variations in the actual and measured positions of the fiducial marker for sinusoidal patterns (a) $\mathrm{A}=20 \mathrm{~mm}$, $\mathrm{T}=$ $2 \mathrm{~s}$; (b) $\mathrm{A}=20 \mathrm{~mm}, \mathrm{~T}=4 \mathrm{~s}$; (c) $\mathrm{A}=40 \mathrm{~mm}, \mathrm{~T}=4 \mathrm{~s}$; and (d) the basic respiratory pattern (mean $\mathrm{A}=15.2 \mathrm{~mm}$, mean $\mathrm{T}=$ $4.8 \mathrm{~s})$.

$=2 \mathrm{~s}), 0.14 \mathrm{~mm}(\mathrm{~A}=20 \mathrm{~mm}, \mathrm{~T}=4 \mathrm{~s})$, and $0.21 \mathrm{~mm}(\mathrm{~A}=40 \mathrm{~mm}, \mathrm{~T}=4 \mathrm{~s})$ for sinusoidal and $0.79 \mathrm{~mm}$ for the basic respiratory pattern, respectively.

\section{Discussion}

In this study, QA was performed to achieve the respiratory-gated RT using the RTRT system. We demonstrated that the respiratory-gated RT using the RTRT system was performed with high dosimetric and geometric accuracy.

Keall et al. reported a QA procedure for quantifying both the dosimetric and geometric accuracy of respiratorygated intensity modulated radiotherapy (IMRT) [13]. Ionization chamber measurements were performed for the stationary, gating and free breathing with the lung IMRT phantom. The relative dose ranged from 0.99 to 1.03 for IMRT fields. Our study demonstrated similar results. When the amplitude was large for sinusoidal (A = 40 $\mathrm{mm}$ ), the relative dose for gating was larger than that for non-gating. Respiratory-gated RT using the RTRT reduced the effect of large respiratory motion on dose delivery accuracy.

Dose profile measurement was performed using Gafchromic films. For sinusoidal and basic respiratory patterns, the differences in lateral distance between the $90 \%$ dose of dose profiles for gating were smaller than those for non-gating. The differences for gating were $\leq 1.8 \mathrm{~mm}$. Saw et al. reported that differences in isodose levels between the measured and planned dose distribution should be within $3.0 \mathrm{~mm}$ in the high-dose region using respiratory-gated IMRT delivery [7]. The International Commission on Radiation Units and Measurement report 42 has recommended that the distance-to-agreement for the high dose region was $\leq 2.0 \mathrm{~mm}$ [14]. Our results were less than those values. Respiratory gated RT using the RTRT system reduced the blurring effect for respiratory motion with high accuracy.

In general, the respiratory gating system is used based on the external surrogate signal [15]-[17]. In external gating, the tumor position is derived using the external breathing signal. The external gating window corresponds to a segment of the external respiratory signal where we turn on the treatment beam. Therefore, the gating feedback mechanism must be able to detect the external surrogate signal. However, the RTRT system based on an internal surrogate signal is used routinely at our institution. According to AAPM TG76, it must be assumed that the tracking accuracy of the internal fiducial marker is more robust than the external signal [18]. Therefore, it is important to verify the tracking accuracy in the QA protocol. For the sinusoidal and basic respiratory pat- 
terns, the RMS values of tracking error were $\leq 1 \mathrm{~mm}$, respectively. Shirato et al. reported that the tracking accuracy of the RTRT system was $\leq 1.5 \mathrm{~mm}$ for a moving target up to a speed of $40 \mathrm{~mm} / \mathrm{s}$ [6]. Our study has demonstrated similar results.

A time delay in a respiratory gating system could cause unexpected phase mismatch for respiratory-gated RT; thus, geometric and dosimetric errors might occur [19] [20]. However, the time delay between recognition of the marker position and the start or stop of dose delivery was $0.03 \mathrm{~s}$ for respiratory-gated RT using the RTRT system [21]. Our QA results showed high dosimetric and geometric accuracy for respiratory-gated RT using the RTRT system. The delay time was clinically acceptable.

The current study was performed under regulated conditions. We verified the position of the moving target only in the SI direction. Given the small motion amplitude and regularity of respiratory patterns in the left-right directions and anterior-posterior directions, however, the dosimetric and geometric errors in directions other than the SI would be either equal to or smaller than those in the SI direction.

\section{Conclusion}

We suggested a QA procedure for respiratory-gated RT using the RTRT system and confirmed that this system substantially reduced motion-induced marginal blurring in dose distribution with high dosimetric and geometric accuracy.

\section{Acknowledgements}

This research was supported by Grant-in-Aid for Young Scientist (B) from the Ministry of Education, Culture, Sports, Science, and technology, Japan (Grant No. 24791294).

\section{References}

[1] Mah, D., Hanley, J., Rosenzweig, K.E., Yorke, E., Braban, L., Ling, C.C., Leibel, S.A. and Mageras, G. (2000) Technical Aspects of the Deep Inspiration Breath-Hold Technique in the Treatment of Thoracic Cancer. International Journal of Radiation Oncology, Biology, Physics, 48, 1175-1185. http://dx.doi.org/10.1016/S0360-3016(00)00747-1

[2] Rosenzweig, K.E., Hanley, J., Mah, D., Mageras, G., Hunt, M., Toner, S., Burman, C., Ling, C.C., Mychalczak, B., Fuks, Z. and Leibel, S.A. (2000) The Deep Inspiration Breath-Hold Technique in the Treatment of Inoperable NonSmall-Cell Lung Cancer. International Journal of Radiation Oncology, Biology, Physics, 48, 81-87. http://dx.doi.org/10.1016/S0360-3016(00)00583-6

[3] Berson, A.M., Emery, R., Rodriguez, L., Richards, G.M., Ng, T., Sanghavi, S. and Barsa, J. (2004) Clinical Experience Using Respiratory Gated Radiation Therapy: Comparison of Free-Breathing and Breath-Hold Techniques. International Journal of Radiation Oncology, Biology, Physics, 60, 419-426. http://dx.doi.org/10.1016/j.ijrobp.2004.03.037

[4] Ford, E.C., Mageras, G.S., Yorke, E., Rosenzweig, K.E., Wagman, R. and Ling, C.C. (2002) Evaluation of Respiratory Movement during Gated Radiotherapy Using Film and Electronic Portal Imaging. International Journal of Radiation Oncology, Biology, Physics, 52, 522-531. http://dx.doi.org/10.1016/S0360-3016(01)02681-5

[5] Ramsey, C.R., Cordrey, I.L. and Oliver, A.L. (1999) A Comparison of Beam Characteristics for Gated and Nongated Clinical x-Ray Beams. Medical Physics, 26, 2086-2091. http://dx.doi.org/10.1118/1.598723

[6] Shirato, H., Shimizu, S., Kunieda, T., Kitamura, K., van Herk, M., Kagei, K., Nishioka, T., Hashimoto, S., Fujita, K., Aoyama, H., Tsuchiya, K., Kudo, K. and Miyasaka, K. (2000) Physical Aspects of a Real-Time Tumor-Tracking System for Gated Radiotherapy. International Journal of Radiation Oncology, Biology, Physics, 48, 1187-1195. http://dx.doi.org/10.1016/S0360-3016(00)00748-3

[7] Saw, C.B., Brandner, E., Selvaraj, R., Chen, H., Saiful Huq, M. and Heron, D.E. (2007) A Review on the Clinical Implementation of Respiratory-Gated Radiation Therapy. Biomedical Imaging and Intervention Journal, 3, e40. http://dx.doi.org/10.2349/biij.3.1.e40

[8] Cardenas, A., Fontenot, J., Forster, K.M., Stevens, C.W. and Starkschall, G. (2004) Quality Assurance Evaluation of Delivery of Respiratory-Gated Treatments. Journal Applied Clinical Medical Physics, 5, 55-61. http://dx.doi.org/10.1120/jacmp.v5i3.2002

[9] Klein, E.E., Hanley, J., Bayouth, J., Yin, F.F., Simon, W., Dresser, S., Serago, C., Aguirre, F., Ma, L., Arjomandy, B., Liu, C., Sandin, C. and Holmes, T. (2009) Task Group 142 Report: Quality Assurance of Medical Accelerators. Medical Physics, 36, 4197-4242.

[10] http://www.cirsinc.com/products/modality/18/dynamic-thorax-phantom/

[11] Shi, C., Papanikolaou, N., Yan, Y., Weng, X. and Jiang, H. (2006) Analysis of the Source of Uncertainty for EDR2 
Film-Based IMRT Quality Assurance. Journal Applied Clinical Medical Physics, 7, 1-8. http://dx.doi.org/10.1120/jacmp.v7i2.2230

[12] Li, X., Yang, Y., Li, T., Fallon, K., Heron, D.E. and Huq, M.S. (2013) Dosimetric Effect of Respiratory Motion on Volumetric-Modulated Arc Therapy-Based Lung SBRT Treatment Delivered by True Beam Machine with Flattening Filter-Free Beam. Journal Applied Clinical Medical Physics, 14,195-204. http://dx.doi.org/10.1120/jacmp.v14i6.4370

[13] Keall, P., Vedam, S., George, R., Bartee, C., Siebers, J., Lerma, F., Weiss, E. and Chung, T. (2006) The Clinical Implementation of Respiratoy-Gated Intensity-Modulated Radiotherapy. Medical Dosimetry, 31, 152-162. http://dx.doi.org/10.1016/j.meddos.2005.12.002

[14] ICRU (1987) Use of Computers in External Beam Radiotherapy Procedures with High-Energy Photons and Electrons. International Commission on Radiation Units and Measurement (ICRU), Report 42.

[15] Cardenas, A., Fontenot, J., Forster, K.M., Stevens, C.W. and Starkschall, G. (2004) Quality Assurance Evaluation of Delivery of Respiratory Gated Treatments. Journal Applied Clinical Medical Physics, 5, 55-61. http://dx.doi.org/10.1120/jacmp.v5i3.2002

[16] Jiang, S.B., Wolfgang, J., Forster, K.M. and Mageras, G.S. (2008) Quality Assurance Challenges for Motion-Adaptive Radiation Therapy: Gating, Breathing Holding, and Four-Dimensional Computed Tomography. International Journal of Radiation Oncology, Biology, Physics, 71, S103-S107.http://dx.doi.org/ 0.1016/j.ijrobp.2007.07.2386

[17] Giraud, P. and Houle, A. (2013) Respiratory Gating for Radiotherapy: Main Technical Aspects and Clinical Benefits. International Scholarly Research Notices, 2013, 1-13. http://dx.doi.org/10.1155/2013/519602

[18] Keall, P.J., Mageras, G.S., Balter, J.M., Emery, R.S., Forster, K.M., Jiang, S.B., Kapatoes, J.M., Low, D.A., Murphy, M.J., Murray, B.R., Ramsey, C.R., Van Herk, M.B., Vedam, S.S., Wong, J.W. and Yorke, E.(2006) Task Group 76 Report: The Management of Respiratory Motion in Radiation Oncology. Medical Physics, 33, 3874-3900.

[19] Jin, J.Y. and Yin, F.F. (2005) Time Delay Measurement for Linac Based Treatment Delivery in Synchronized Respiratory Gating Radiotherapy. Medical Physics, 32, 1293-1296.http://dx.doi.org/10.1118/1.1896452

[20] Smith, W.L. and Becker, N. (2009) Time Delays and Margins in Gated Radiotherapy. Journal Applied Clinical Medical Physics, 10, 140-154. http://dx.doi.org/10.1120/jacmp.v10i3.2896

[21] Shirato, H., Shimizu, S., Kitamura, K., Nishioka, T., Kagei, K., Hashimoto, S., Aoyama, H., Kunieda, T., Shinohara, N., Dosaka-Akita, H. and Miyasaka, K. (2000) Four-Dimensional Treatment Planning and Fluoroscopic Real-Time Tumor Tracking Radiotherapy for Moving Tumor. International Journal of Radiation Oncology, Biology, Physics, 48, 432-442. http://dx.doi.org/10.1016/S0360-3016(00)00625-8. 
Scientific Research Publishing (SCIRP) is one of the largest Open Access journal publishers. It is currently publishing more than 200 open access, online, peer-reviewed journals covering a wide range of academic disciplines. SCIRP serves the worldwide academic communities and contributes to the progress and application of science with its publication.

Other selected journals from SCIRP are listed as below. Submit your manuscript to us via either submit@scirp.org or Online Submission Portal.
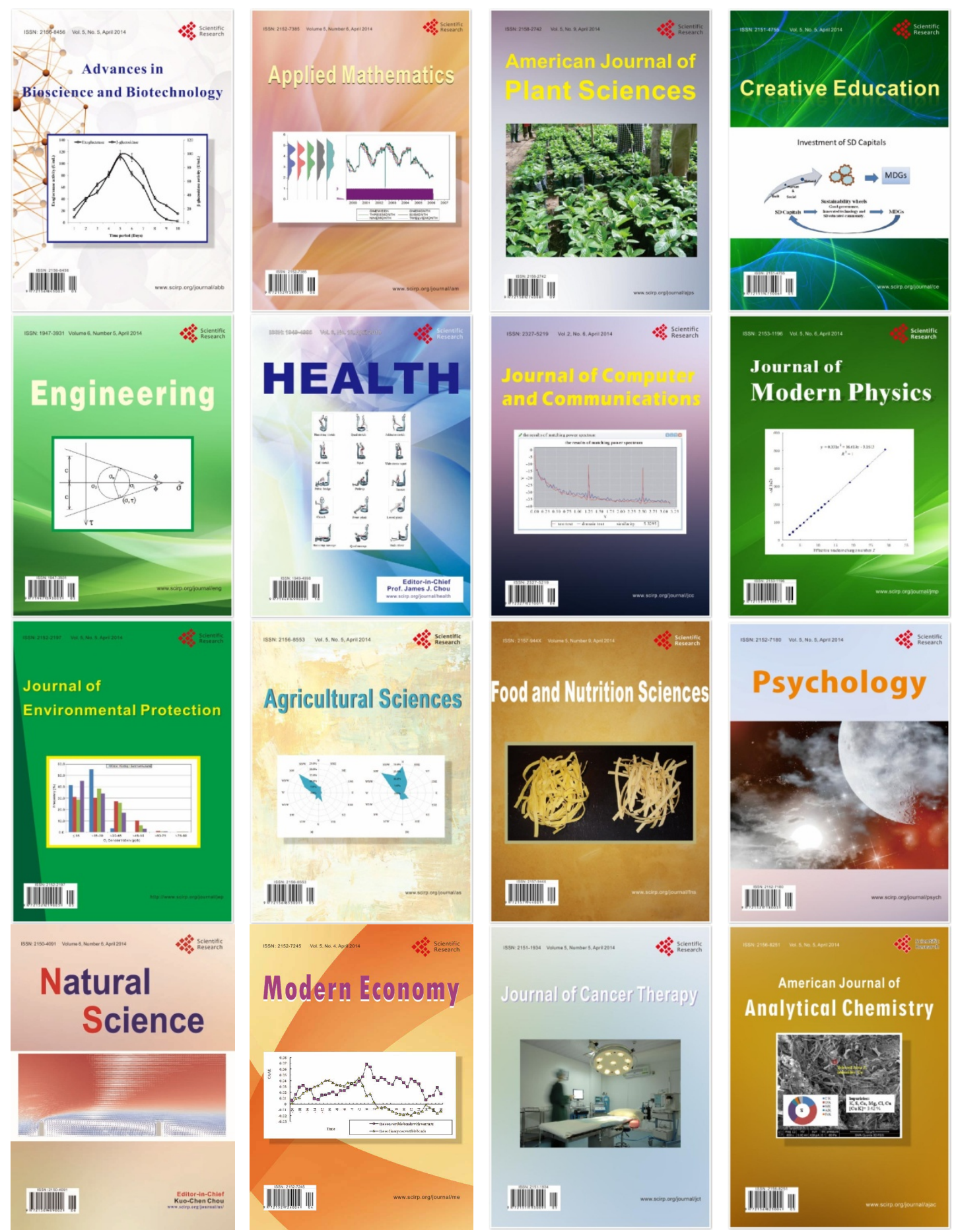